\title{
NEW AIR TRAFFIC MANAGEMENT CONCEPTS ANALYSIS METHODOLOGY: APPLICATION TO A MULTI-SECTOR PLANNER IN US AIRSPACE
}

\author{
Kevin Corker, San Jose State University, San Jose, Ca. \\ Diana Liang, Federal Aviation Administration, Washington, DC \\ Paul Lee, San Jose State University, San Jose, Ca. \\ Tom Prevot San Jose State University, San Jose, Ca.
}

\begin{abstract}
${ }^{1}$
Several developments in the technologies supporting air traffic management (ATM) such as digital communication and improved positioning accuracy for aircraft, have enabled consideration of new organizational and functional operations. One such consideration is a modification of the standard air traffic control team to include a mutli-sector planner (MSP) position. This MSP is being investigated in several research and field studies both in Europe and in the U.S. The feasibility and effectiveness of two of these concept variations was investigated in the current study. The experiment consisted of a pair of one-week human-n-the-loop studies in which the two concepts (Multi-D, in which multiple R-sides are supported by a single D side, and Area Flow, in which the MSP manages flow through their target sector by coordinating with adjacent MSPS., were tested separately with two different 5-person teams, A baseline condition which assumed traditional radar-data roles but with access to advanced decision support tools was also run. Overall, the data suggest feasibility of both variations in the MSP. Workload was manageable for the MSP operations. Area flow operations were found to coordinated with advanced air traffic operations concepts and were shown to be acceptable to the controllers (both $\mathrm{R}$ and $\mathrm{D}$ side)>
\end{abstract}

\section{Advanced Airspace Concepts}

There are an expanding number and variation of airspace operational concepts that are under development and prototype implementation in the US and Europe sponsored by the international programmes: One EATM, SESAR, Next Generation Air Transportation System (NextGen) As a result there is increasingly urgent need for a methodology to evaluate the concepts and their

\footnotetext{
${ }^{1}$ The work reported here was supported by Federal Aviation Administration

ATO - Ops Planning Systems Engineering
}

variations, as they operate not only in isolation but also in conjunction with other developing concepts and in a manner consistent with the over all system development runtime and resource process. The visions assert new modes of operation and technological requirements. Essentially without exception these technologies fundamentally change the process of the work of air traffic and air space management. The "advancements" include a redistribution of information and control among the humans and the automation systems in airspace operations that alters decision modes, execution modes and optimization processes among all participants in the aerospace transportation process. We feel that the changes in the work of air transportation operations require an approach to analysis that includes concern for the change in the cognitive processes that supports the work in context.

The work described here provides a method that comprehensively deals with the assessment of the operational feasibility of concept elements, embeds these in a larger system operational assessment and explicitly concerns itself with transition to the human and system as these operational services are provided. We apply this method to a common "next step" in air navigation service provision, the "multi-sector planner". (MSP)

\section{Multi-Sector Planner}

Air traffic control in the en route airspace environment in the United States (U.S) has traditionally been performed by a team, consisting of a radar-controller (R-side) and a second controller referred to as a data-controller (D-side) or a radar-associate. Several developments in the technology supporting air traffic management digital data communication among controllers and between controllers and aircraft, improved positioning accuracy for flight operations, conflict prediction, and sector complexity assessment have enabled consideration of new organizational 
structure and functional standards for team operations. One such consideration is a modification of a standard configuration to include a "multi-sector planner" (MSP) position in the team. This MSP position has been investigated in several research and field studies, both in the U.S. and in Europe. ${ }^{2}$ The concept provides a spectrum of redistributed roles and responsibilities among the air traffic management team members including physical relocation. The feasibility and effectiveness of two variations of these concepts were investigated initially with the Cognitive Systems Engineering evaluative methodology described in the following section.

\section{Cognitive Systems Engineering (CSE)}

The purpose of this paper is twofold. First, we intend to offer an evaluative methodology that we think has general utility and validity in today's Air Traffic development environment for multiple systems. Second, we demonstrate application of that methodology in evaluation of a target concept of operations, the MSP. The MSP process includes common elements for all such ATM advances: technology advance and pull, capacity and resource based demand, human operators with current and changing roles and significant technical-political development advocacy.

\section{ATM systems as joint cognitive systems}

When evaluating future ATM systems, it is meaningful to consider the humans and technologies as a single unit of analysis [2]. In other words, it is the system as a whole that is considered capable of cognitive work such as setting goals, assessing the state of the world, affecting change, modifying goals, managing feedback, and exerting control. There are several implications for taking this approach. More traditional approaches to human factors have been concerned with information gathering, assessment, and communication. One component of CSE is indeed to study and design how information and knowledge is gathered, maintained, and passed among the various agents within the system. Complementary to this, CSE is also concerned with overall system qualities, such as robustness to perturbations, its ability to deal with novel and unique circumstances, the dynamics of the system as a whole, its ability to scale up, the distribution of work across agents within the system, and the communication between those agents. The

\footnotetext{
${ }^{2}$ A full past and ongoing research is provided in the full experiment report [1] and is available either at the SJSU HAIL website: www.sjsuhail.org: or will be provided at an email request
}

approach recognizes the importance of the environments in which a number of cognitive agents (human or machine) are immersed [3, 4].

The example at hand, the MSP evaluation process raises several issues associated with concern for the human-system integration and its potential disruption in new system deployment. These are summarized as follows:

- Underspecified future operations

- Incorporation of prior best practice

- Performance expectations

\section{Underspecified Future Operations}

Each view of the future use of the MSP is underspecified in terms of the impact that the proposed technology will have on practice within a system. The focus is on system functionality, procedures, and performance measurements, system status measurements (workload, amount of communication, and similar measurements). Rarely is the impact that the new technology will have on the constraints that a system operates taken under much of a consideration, yet this can lead to powerful insights in terms of how well the investment will succeed.

\section{Incorporation of Prior Best Practice}

The proposed designs are often ungrounded. Many times they fail to incorporate what practitioners in the existing system have learnt from current practice; many times they also fail to take into considerations what has been learnt by the human factors community about cognition in complex, industrial systems.

\section{Performance Expectations}

A final characteristic of future operations is that expectations are poorly calibrated: very often, they are overly optimistic in that the envisioned (performance) benefits will be reached and that they are considered the only impact that the investment will have.

We have explicitly addressed these issues in our cognitive engineering analysis.

\section{MSP Cognitive Engineering Analyses}

The MSP is a concept that changes the fundamental team structure for standard en route air traffic control. As such, it is sensitive to the context of operations in which it is implemented. In keeping with our CES approach, we undertook four analyses prior to the development of our experimental design for human-in-the-loop 
simulation. These analyses included both operations in the US and in Europe.

\section{Analysis One: Information sharing}

A workshop was held at the William J. Hughes Technical Center in Atlantic City, New Jersey in October 2003 in order to establish an operational definition of the MSP process. The workshop included researchers from NASA, FAA, MitreCAASD, and universities. The outcome of this meeting was an initial concept of operations for the MSP in US airspace.

\section{Analysis Two: Definition of roles and responsibilities and information requirements}

Armed with the FAA's initial prototype concept definitions for MSP, a comprehensive literature review was completed. European researchers involved in MSP concept feasibility analyses at DFS, EEC, and AENA were interviewed and site visits to their laboratories were undertaken. In addition, three meetings were held in which several operational experts and research scientists (field facility supervisors, en route airspace design engineers, FAA contractors, and NASA researchers) discussed and determined the implications of the FAA's strategic vision for the provision of services on the potential for the successful implementation of MSP. The results of these meetings included a preliminary description of information requirements and the operator roles and responsibilities for both the current and existing operational tools. These were verified by site visits and reviews by a number of field supervisors. The output of this phase is a report by Booz Allen Hamilton [5].

\section{Analysis Three: Cognitive walk-throughs of initial MSP concepts}

Two scenarios were developed which represented different perspectives of the operational concept. In one of the scenarios, a single data controller supported multiple radar controllers. In the other, the data controller served as a multisector coordinator managing traffic flow in the target sector. Cognitive walk-through methods were undertaken with three sets of five controllers. The outcome of these analyses (ref SJSU report) established task allocation and coordination requirements.

\section{Analysis Four: Human-in-the-loop laboratory capabilities}

Site visits and surveys of US and European laboratory facilities were conducted. The facilities were visited and the capabilities of the laboratories were evaluated as to the suitability for MSP research based upon the outcome of the cognitive walk-throughs. In addition, availability and schedule were considered. Based upon that evaluation, the NASA Ames Airspace Operations Laboratory (AOL) [6] was selected as the site of the human-in-the-loop MSP study.

\section{Human-in-the-loop Experimental Conditions}

In our experiment, one variation of the MSP concept, termed "Multi-D", took the traditional role of a data-controller but provided these types of services to several radar controllers (three radar controllers were assigned to be the responsibility of the data-controller in this study). As in current operations, the radar position had the responsibility for managing the sector operations for individual sectors, including aircraft separation and traffic flows. The Multi-D position supported the R-side by managing traffic flows within the multi-sectors and providing medium-term conflict resolutions, as well as assuming normal data-controller duties but with advanced automation assistance. Multi-D was provided with a traffic situation display that spanned across three sectors, a conflict probe with approximately 15-minute look-ahead time along the aircraft 4D trajectory, route/altitude trial plan capability, ground-to-air data link to uplink clearances to the flight crew, ground-to-ground data link to coordinate route/altitude modifications among ground stations, sector load graphs and table, electronic flight strips, and "quick look" capability to look at an individual sector.

In the second configuration, the MSP served functions often associated with traffic flow management, coordinating with external MSP areas and attempting to manage sector traffic levels in a proactive process, balancing loads among the three sectors in their area of responsibility as well as with external areas. This function was termed "Area Flow Planner". In this MSP role, the Area Flow was meant to be a bridge between Traffic Management Unit (TMU) and R-side controllers. Since the role focused on strategic flow issues and did not involve tactical control of operations, the Area Flow was not co-located with the R-side controllers in the study. Unlike Multi-D, Area Flow did not resolve medium-term conflicts. Instead, s/he actively managed the sector loads across the three sectors by rerouting aircraft to keep the aircraft count below the Monitor Alert Parameter (MAP). Except the conflict probe and ground-to-air data link capability, the Area Flow position was equipped with same tools as the Multi-D. 
The experiment consisted of a pair of oneweek human-in-the-loop studies, in which the two MSP concepts (i.e. Multi-D and Area Flow) were tested separately with a different team, each consisting of five participants. The MSP position provided services for three radar-controllers in a modified Forth Worth center airspace. A "ghost MSP” position was staffed by one of the participants to act as an adjacent MSP, so that coordination activities between MSPs could be captured within the study. The participants were presented with two different types of scenarios, a high traffic scenario without weather and a moderate traffic scenario with weather, that were designed to exercise different facets of an MSP's roles and responsibilities. In addition to running one of two MSP conditions (i.e. Multi-D or Area Flow) in each week, a baseline condition - in which two of the three sectors were staffed with radar and data-controller pairs, and the third with an R-side only - was also run. This allowed examination of the team's performance in a baseline condition versus one of the experimental conditions. The baseline condition assumed maintenance of the current day team concept of radar and data-controllers but with the presence of advanced decision support tools and automation, such as data link, conflict probe, and 4D trajectory trial planning capability. An equivalent set of advanced tools in both MSP and the Baseline conditions means that significant differences in the results indicate the impact of the shift in roles and responsibilities that resulted from the MSP concepts. Those tools are described $\mathrm{n}$ detail in [1, 7]

\section{Experimental design}

The use of the CSE approach in the earlier analyses of the MSP provides economies and efficiencies in human-in-the-loop (HITL) experimental design. We took advantage of those efficiencies. For instance, we did not use a baseline of current day operations with current day technologies as we knew that other groups were specifically targeting such capacity issues [7, 8, and 9]. We were able to focus the scenarios to stress the information and coordination issues that were identified in our earlier cognitive walkthrough. We were also able to focus the data that were collected and analyzed to be specifically sensitive to matters of roles and responsibilities that had been identified in the CSE process as likely indicators of the concept operational robustness under traffic and weather perturbations.

\section{Goals}

The overall goal of this study was to examine two relatively polar concepts of multi sector planner in the context of US air traffic management. The study had nested purposes. The first was to determine, given a suite of tools and procedures, if the operational concept in either of its two forms is feasible in US operations. To answer this question, the experiment compared the performance of the MSP concepts against a baseline procedural concept that included the advanced tools of a future ATM, but with no change in the roles, responsibilities, and team structure.

The second purpose was to attempt to identify the relative strengths of the two forms of the operational concepts being examined with respect to efficiency and throughput. This latter purpose must take into account varying operational demands and constraints that might favor or challenge one form of MSP concept or the other. The basic approach would be to test the two implementations of the MSP concept (Multi-D \& Area Flow) against a set of traffic and environmental conditions. That comparison can be made with some constraints in the strength of the inferences that can be drawn.

\section{Constraints in the Experimental Design}

An independent examination of the two MSP concept variants (i.e., Multi-D and Area Flow) in two separate one-week studies with different participants was necessary to assure that the roles and responsibilities assigned to one concept did not cross over to the other. This between-subject design of the two MSP concepts meant that the results from Multi-D and Area Flow conditions were not directly comparable. For example, if the Area Flow planners rated the acceptability of the concept higher relative to Multi-Ds, one could not infer that Area Flow concept was more acceptable since the differences could easily be due to individual differences (e.g. personal bias, aptitude, etc.). To address this problem, the results were compared within-subject whenever possible. In particular, comparisons between baseline and MSP conditions during the same week were used heavily as they provided the most reliable results. When comparisons were needed across the participant teams (i.e. Multi-D vs. Area Flow participants), the data from the baseline conditions between the two participant teams were compared first since baseline conditions were identical across the weeks. If significant differences occurred, they would be attributed to individual differences between the teams. However, if there were no differences for the baseline data, then any differences between Multi-D and Area Flow results could be better trusted to have resulted from conceptual differences. During the data analyses, these constraints and factors were considered to ensure that data analyses and subsequent conclusions from 
the data were not erroneous due to these other factors. Figure 1. illustrates this process.

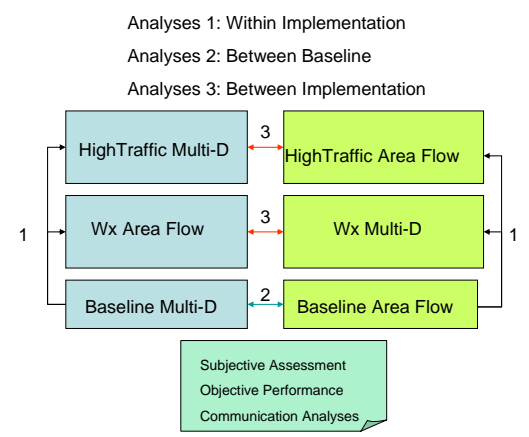

\section{Results}

The feasibility and operational benefits of the two MSP concepts were investigated first by comparing within-subject performance data - i.e. performance of subject participants operating under each MSP operations were compared against the performance of the same participants in the baseline condition. These within-subject analyses examined the relative benefits and shortcomings of each MSP concept compared to a more traditional $\mathrm{R}$ and $\mathrm{D}$ controller team without potential confounds due to individual differences.

\section{Measures}

In this abbreviated summary of the research, we are unable to provide the full set of results from the experiment. However, a brief summary of the measures taken (and available in full in [1]) is described below:

Separation violations - Separation violations were recorded whenever two aircraft were within 5 nm lateral and $\pm 1,000$-foot vertical buffer.

Late conflict resolution - The system can be set up to record the conflict probe event, which is triggered whenever the automation detects a conflict along the 4D trajectory. A dedicated data collection station recorded all conflict probes that were not resolved with less than 5 minutes until the loss of separation across all sectors.

Aircraft count - Aircraft count was measured to understand the traffic load and the associated controller workload.

Number of tactical maneuvers - In this study, the controller participants used the advanced trial planning capabilities and route uplink via data link whenever, and as early as possible. Remaining maneuvers were tactical vectors and altitude changes issued by voice.
Number of clearances - Similar to the tactical maneuvers, all clearances can also be extracted from the pseudo-pilot stations

ETA's to the destination airport - estimated time of arrivals (ETAs) are calculated and logged periodically by the simulation software

Sector transit time - Sector transit time is captured by logging the time when an aircraft enters and exits a sector.

WAKS - Workload Assessment Keyboard Scale gathers workload ratings from participants in real time.

CARS - Lee, Kerns, Bone \& Nickelson (2001) as a derivative of the Cooper-Harper Rating Scale, developed the "Controller Acceptance Rating Scale.”

NASA TLX - The Task Load Index was developed at NASA Ames Research Center by Hart \& Staveland (1988) to measure workload experienced over a period of time, such as summarizing workload over a simulation run.

Number of coordination's among the controllers - This measure identified the number and the types of coordination between the members of the participant team.

Number of cancelled trial plans - This measure identified the number of trial plans that were started but abandoned before they were coordinated.

Number of trial plan coordination and uplinks - This measure identified which one of the concepts generated and executed more trial plans by the MSP or the corresponding D-side positions.

Duration of trial planning - This measure identified the average amount of time a participant either the MSP/G-MSP in the MSP condition or the D-sides in the Baseline - would spend on trial planning.

Weather penetrations - Under the weather conditions, the controller participants attempted to maneuver aircraft around weather cells.

\section{Airspace}

The simulation airspace was a modified DallasFort Worth Center (ZFW) airspace. Controller participants worked the three high-altitude sectors Wichita Falls (SPS), Ardmore (ADM), and Decod (DECOD). In this study, all three sectors were expanded to increase sector size and complexity (see Figure 2). Wichita Falls and Decod were expanded to include departure streams in sectors 94 and 90, respectively, so that the controller participants would manage both arrivals and 
departures in these sectors. Ardmore sector also increased its size to allow the controller to have more maneuver room for the southbound arrivals.

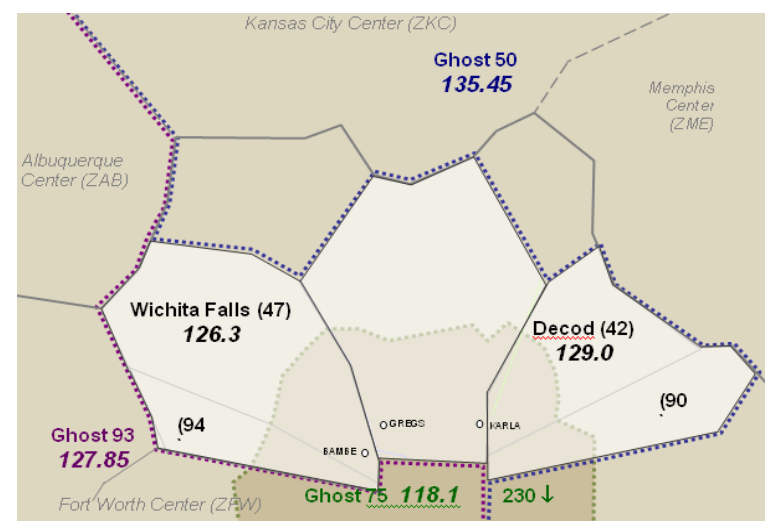

Figure 2. Airspace for HITL opérations

\section{Data}

\section{Aircraft Count}

The traffic scenarios were designed to create peak traffic in each sector that would require D-side or MSP's assistance to be able to manage the traffic. In non-weather scenarios, Falls and Ardmore sectors peaked above 20 aircraft for about 15 minutes at 30 and 45 minutes into the scenario, respectively. Decod, which did not receive any Dside assistance in the baseline condition, had peak traffic at 18 aircraft at about 30 minutes into the scenario. The weather scenarios had similar overall patterns but the peak counts were reduced to about 16 aircraft.

Figure 3.a,b,c illustrates the actual aircraft count that each controller managed during baseline and Multi-D operations. In this figure, the aircraft count data were collapsed for weather and nonweather scenarios for simplicity. The graphs confirmed that the overall traffic pattern remained the same after controllers managed them as when they were originally set up.

More importantly, the aircraft count and the overall traffic pattern remained the same between baseline and MSP conditions in Multi-D operations. Although not shown here, these patterns held true for both weather and non-weather scenarios. This result suggests that Multi-D controller did not significantly redirect the traffic out of the test sectors during the simulation runs. Similar aircraft count between baseline and MSP ensures that any potential differences in other metrics (e.g. workload, number of conflicts, etc.) did not result simply from controllers managing fewer aircraft.

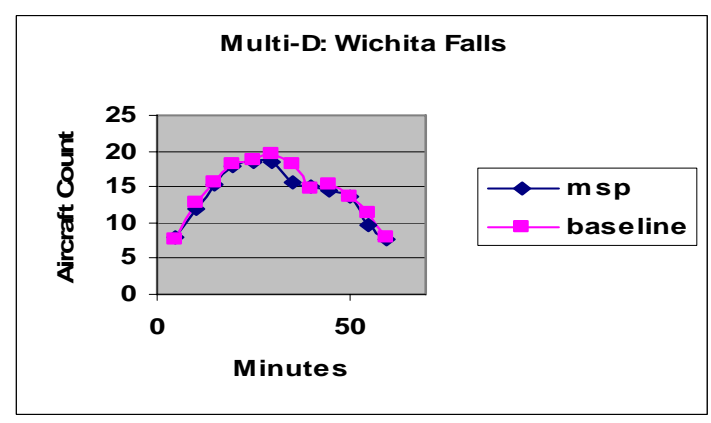

Table 3 a: Wichita Falls Traffic

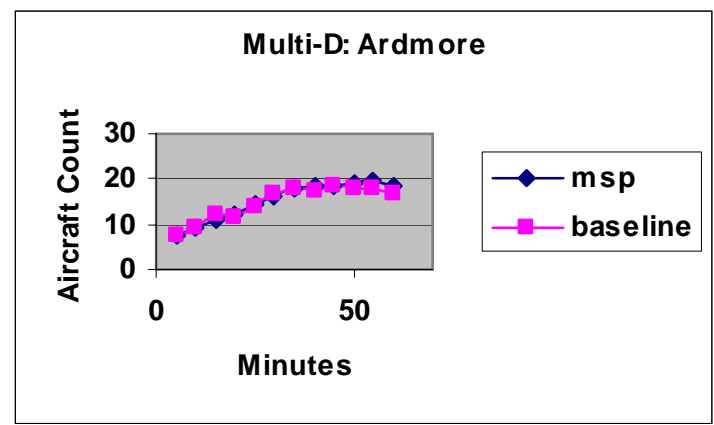

Table 3 b: Ardmore Traffic

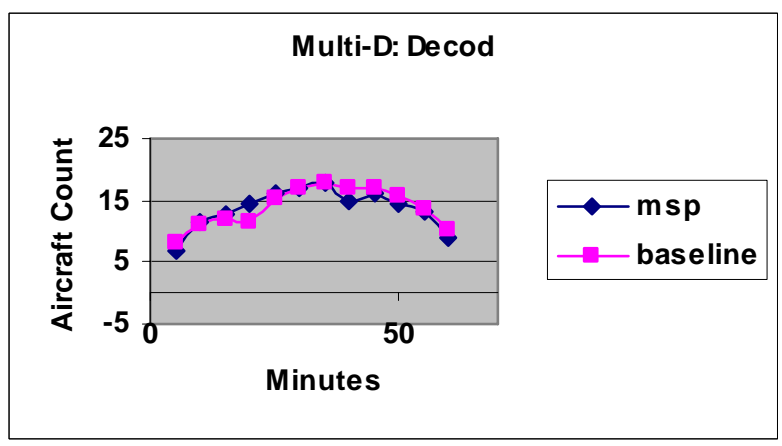

Table 3 c: Decod Traffic

\section{ETAs to the Destination Airport}

Table 3.1.2.1 shows the delay (in seconds) that each aircraft incurred during the simulation. Since aircraft were rerouted around weather and to manage traffic, all conditions resulted in delays.

In weather scenarios, MSP condition resulted in less delay. In non-weather scenarios, baseline resulted in less delay. Both results were only marginally significant.

Table 1. Delays (in seconds) per Aircraft in Multi-D Operations 


\begin{tabular}{|l|c|c|c|c|}
\hline \multirow{2}{*}{ Scenarios } & \multicolumn{3}{|c|}{ Condition } \\
\cline { 3 - 5 } & MSP & Baseline & $\begin{array}{c}\text { MSP - } \\
\text { Baseline }\end{array}$ \\
\hline \multirow{2}{*}{$\begin{array}{l}\text { Multi- } \\
\text { D }\end{array}$} & Weather & 68.4 & 100.4 & -32.0 \\
\cline { 2 - 5 } & $\begin{array}{c}\text { High } \\
\text { Traffic }\end{array}$ & 40.9 & 20.6 & 20.3 \\
\hline
\end{tabular}

High traffic $\mathrm{p}<0.15$; Wx, $\mathrm{p}<0.07$

Table 1. Delays compared to ETA in standard and weather operations.

Table 2 shows combined results of low and high intensity weather data operations with the dependent variable being weather penetrations. The overall results show fewer weather penetrations for Ardmore sector under MSP condition $(\mathrm{n}=41)$ compared to baseline $\left(n=62 ; \chi_{1}^{2}=4.3, p<0.04\right)$, suggesting that Multi-D position may have helped Ardmore controller in weather avoidance maneuvers.

Table 2 Total Weather Penetrations for Multi-D Operations

\begin{tabular}{|l|c|c|c|c|}
\hline \multirow{2}{*}{} & \multirow{2}{*}{ Condition } & \multicolumn{3}{|c|}{ Test Sectors } \\
\cline { 3 - 5 } & $\begin{array}{c}\text { Wichita } \\
\text { Falls }\end{array}$ & Ardmore & Decod \\
\hline \multirow{2}{*}{$\begin{array}{l}\text { Multi- } \\
\text { D }\end{array}$} & Baseline & 0 & $62^{*}$ & 12 \\
\cline { 2 - 5 } & MSP & 0 & $41^{*}$ & 10 \\
\hline
\end{tabular}

* Results are significant at $\mathrm{p}<0.05$.

\section{Subjective Workload Assessment}

Figure 4 shows the AF/D-side controller's WAKs ratings for a scenario in which there is an increase in traffic load peaking at about 40 minutes into the scenario (represented by data sample 7). The D-side, reported lower workload initially under the baseline conditions rising to a medium level of workload toward the end of the scenario - a pattern similar to that reported by the R-side controller, although it does not rise quite as early. However, when this participant was in the AF position, he reported higher workload initially in the scenario, dropping down toward the end, although the higher workload was no higher than in the D-side position, just timed differently.

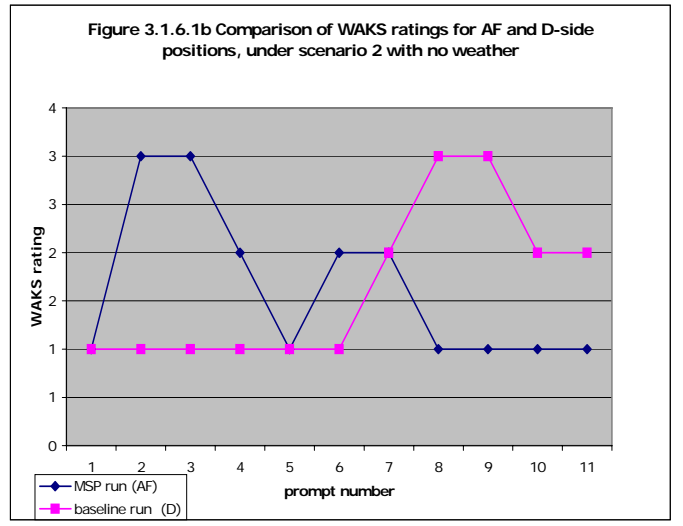

Figure 4: Workload estimates in 5-minute increrements,

\section{Summary of Results}

We will summarize the results of the study according to operational performance categories of strategic versus tactical control modes, efficiency, safety, and controller focused categories of workload, communication and coordination and acceptability.

\section{Multi-Sector Planner: Strategic versus Tactical Control}

One goal of re-distributing the roles and responsibilities in the MSP is to attempt to gain a broader cross-sector view of control and the impact of maneuvers. The results indicate that the MSP operation (in either form) does contribute to a more strategic mode of operation. This is indicated by a significant decrease in the number of "late" maneuvers to avoid conflict in high traffic operations managed by the Multi-D. In both Multi$\mathrm{D}$ and Area Flow operations there was a significant reduction of severe weather penetrations.

\section{Multi-Sector Planner: Safety of Operations}

It has been noted in several European studies of an MSP concept similar in structure to the Multi$\mathrm{D}$ operations in our study that there was a subjective concern expressed by the controllers at the loss of a person-to-person back-up to identify and avoid conflict $[7,8,9])$. In our study, the number of operational errors/separation violations did not suggest any change in objective safety between the concepts or between the concepts and the baseline operations. From a subjective perspective, the R-side controllers thought the Multi-D operations were as safe as the Baseline 
while the Multi-D controllers thought that the safety was slightly compromised due a reduction in situation awareness compared to D-side. For Area Flow, there were fewer separation violations compared to Baseline. Furthermore, both R-side controllers and Area Flow planners thought that the Area Flow operations were safer than the Baseline operations. It is worth noting that the controllers felt that operations were generally safe overall, though the MSPs reported that they did not (and could not) maintain situational awareness of all the sectors' traffic in traditional terms.

\section{Multi-Sector Planner: Efficiency of Operations}

The efficiency of operations in MSP modes was roughly equivalent to the baseline operations. The Multi-D provided a reduction of delays in the weather condition. The Area Flow operations did not contribute to significant delays in the weather condition but did contribute to significant delays in the high traffic condition. This maintenance of efficiency is provided despite the reduction of the team staffing by two controllers.

Interestingly, participants rated both Multi-D and Area Flow operations to be more efficient than the Baseline. This was a subjective rating of their experience and referred to their impression of both the traffic flow, as well as the process of control. For example, MSP operations were thought to be more efficient since "key strategic moves by the MSP would remedy problems in multiple sectors".

\section{Multi-Sector Planner: Workload}

Workload analyses indicate the radarcontrollers workload was not increased (and was somewhat decreased in Area Flow operations) in the MSP operations. The MSPs' workload as either Multi-D or Area Flow controllers were increased, but not beyond manageable levels. Overall there was a more equitable balance of workload across the MSP teams than that reported in current R-side, D-side operations.

The analyses of the participant workload suggested that in the Multi-D conditions, the radarcontrollers' workload was relatively unaffected by the conditions (i.e. Multi-D vs. Baseline), suggesting that a Multi-D was as effective in aiding the radar-controllers as the two data-controllers in the Baseline condition.

In the Area Flow condition, the Area Flow planner was able to reduce the radar-controllers' workload by attempting to adhere to specific aircraft count targets for the sectors under his control. The workload for the Area Flow planner mainly consisted of coordinating with the adjacent Area Flow planner to manage the traffic flow far away from the impacted sectors, resulting in frequent verbal coordination between them but only few verbal coordination activities with the radarcontrollers.

\section{Multi-Sector Planner: Communication and Coordination}

An examination of the MSP communication/coordination suggest an expected shift from frequent exchange between R-side and D-side controllers in baseline conditions to less frequent exchange between MSP and R-sides. In the MSP condition, there was an additional coordination process with the adjacent MSPs. Analyses of the route coordination between participants suggest that, in general, Multi-D and Area Flow had fewer coordinations compared to Dsides in Baseline. However, the "ghost" Multi-D and Area Flow had an equal or greater number of coordination events than their counterparts, suggesting that a portion of the coordination efforts were "passed on" to the upstream sectors. The feasibility of such operations, when the upstream sectors also have full traffic levels, needs to be addressed in future studies. Communication strategies, in our opinion, did not have time to evolve to a stable and predictable structure within the time of the experiment. Longitudinal studies of controller coordination and communication with a larger number of participants is recommended in order to better understand the impact of the changes of roles and responsibilities represented by the MSP operational concept.

\section{Multi-Sector Planner: Controller Acceptance of Operational Concept}

Like the studies reported by [7.8] the opinions of the controllers was "guardedly positive". For the Multi-D concept, R-side controllers gave high acceptability ratings to their positions in both Baseline and Multi-D conditions. Multi-D participants gave lower ratings overall for the positions that they staffed. For the Area Flow concept, the participants rated their positions as slightly less acceptable than in the Baseline concept. Although the overall ratings were favorable to Area Flow position, data suggest that Multi-D position showed better acceptability in comparison to their respective Baseline ratings.

As is going to be the case in systems transition, authority and responsibility will need to be tuned through experience. One issue that favors consideration of the Area Flow concept as an appropriate development direction is its rather clear difference from current roles and responsibilities. 
There was a clear tension between the radar controllers and Multi-D with respect to the final authority in a sector. Multi-Ds thought that they had a greater authority to re-direct aircraft based on a larger picture of the traffic situation while the radarcontrollers thought that, as a data-controller, MultiD should maintain a similar level of authority as current day data-controllers.

Area Flow planners have a broader perspective and make decisions that are often managed outside the area of the sectors known to the R-sides, through coordination with adjacent MSPs. (The processes of this coordination will have to be the focus of future analysis and experimentation.) As such, their actions are not subject to review by the R-Side controller. This is a rather more extensive change of roles than the Multi-D. R-sides expressed some confusion as to their responsibility or authority to intervene and modify the Area Flow's plan. This issue was also cited in European studies and analyses of information requirements. It is worth noting, that this confusion, as to the basis for modification of a plan provided by a planner (either human or automation) with a broader and presumably more globally optimal plan is a fundamental issue in the development and implementation of the more advanced NextGen.. The participants also commented that the acceptability of the Area Flow concept predicated on having proper tools to assess and execute traffic flow initiatives, such as accurate departure information, shortcut functions to re-route multiple aircraft along a similar route, and better traffic complexity indicators. As noted in the discussion of communication and coordination, the Area Flow tools did not provide conflict detection (as that was not their intended role). However, Area Flow controllers often used the route planning tool to investigate potential conflicts.

\section{Conclusions}

\section{Feasibility}

The study proved the feasibility of the concept of a Multi-Sector Planner position in en route operations. The study differentiated different mechanisms whereby safety, efficiency, and redistribution of workload can be achieved with two operational instantiations of the concept of MSP. While not intended to definitively distinguish between the two operational concepts, the results have suggested a distinction between Area Flow and Multi-D operations.

\section{Operational Concept Development Direction}

The operational integration differences between the Area Flow and Multi-D operations do suggest that Area Flow provides a more consistent path for future development aligned with Operational Evolution Partnership (OEP) and NextGen development. The change of roles to a planner, and the strategy of reduction of possible conflicts to reduce reliance on tactical response from the D-side provides for a clearer interaction and authority process for the controllers. The Area Flow operations also allows for a more flexible control station configuration as the Multi-D operations did require the Area Flow to be physically co-located with the Radar-controllers in the operations.

The Area Flow operation also could be aided by tools that are consistent with planned NextGen and OEP development, through integration of information and planning for more strategic rerouting. So, given no clear operational cost to Area Flow development, that operational concept is more consistent with future airspace operations development.

\section{Research Requirements}

In pursuit of operational refinement of the Area Flow concept, it is necessary that several significant areas of research be pursued. We have not yet researched the impact of interactions among several MSPs who have areas of responsibility that require active management and coordination while they are also being asked to assist other adjacent MSPs in there area flow. We also have not undertaken a systematic analysis of the roles, responsibilities and procedures required to integrate the operation of multiple Area Flow MSPs with the larger Center control and flow requirements. This then extends to the coordination of center requirements with NAS level requirements.

We also have not undertaken experiments to identify what tools might be required for the Area Flow in their control of traffic in their specific areas and for the yet to be defined coordination requirements with other MSPs. The level of automation and the type of decisions aids that could be used to support MSP operations should be explored.

These analyses and simulations are recommended to advance the development of an Area Flow configuration in the context of ongoing NextGen technical developments. 


\section{References}

[1] Corker, K., Lee, P., Prevot, T., Guneratne, E., Martin, L., Smith, N., Verma, S., Homola, J. \& Mercer, J.(2006). Analysis of Multi-Sector Planner in U.S. Airspace. San Jose State University, HAIL Lab report Mo. 2006_3342_01.

[2] Hollnagel, E. \& Woods, D. D. (1983). "Cognitive Systems Engineering: New wine in new bottles". Int. J. of Man-Machine Studies, 18, 583600.

[3] Flach, J., Hancock, P., Caird, J., \& Vicente, K. (Eds.). (1995). An ecological approach to human machine systems. Lawrence Erlbaum

[4] Flach, J. M. (2000). An ecological approach to human-machine systems. In Proc. IEA 2000/HFES 2000 Congress.

[5] Booz Allen Hamilton (2004). Analysis report of the concept of use for en route modernization. McLean VA:

[6] Prevot, T., N. Smith, E. Palmer, J. Mercer, P. Lee, J. Homola, T. Callantine, 2006, The Airspace Operations Laboratory (AOL) at NASA Ames Research Center, AIAA-2006-6112, AIAA, Reston, VA.

[7] Prevot T. , Lee P.., Martin L., Mercer J., Palmer E., Smith N. (2006) Tools for Trajectory-Based Air Traffic Control and Multi Sector Planning HCIAero’06, September 2006, Seattle,WA

[8] Herr, S., Teichmann, M., Poppe, M., \& Sharez, N. (2003). Gate-to-Gate Key Concept Multi Sector Planning (Version 0.5). Langen, Germany: DFS Deutsche Flugsicherung GmbH.

[9] Garcia-Chico, J. L. et al. (2004). Cluster 2 Operational Concept (En-route and multi-layered planning). (Version 1.0). Spain, Madrid: Gate-toGate Programme.

[10] Lee, K., Kerns, K., Bone, R., \& Nickelson, M. (2001). Development and validation of the controller acceptance rating scale (CARS): Results of empirical research. Proceedings of the $4^{\text {th }}$ USA/Europe Air Traffic Management $R \& D$ Seminar, Santa Fe, NM.

[11] Hart, S. \& Staveland, L. (1988). Development of the NASA-TLX Results of empirical and theoretical research. In P. A., Hancock \& N., Meshkati (Eds.), Human Mental
Workload (pp. 239-250). Amsterdam: North Holland Press.

\footnotetext{
${ }^{3}$ Acknowledgement: The authors would like to thank our colleagues form Mitre-CAASD (Mr. Bolzak, Dr. Celio, Dr, Viets) for their participation, observation and support. We would also like to thank all participant controllers for the hard work and excellent feedback.
} 
- 11 - 